\title{
Psychopharmaka in der Schwangerschaft
}

Da viele psychiatrische Erkrankungen im jungen Erwachsenenalter manifest werden, gibt es auch immer mehr Frauen im gebärfähigen Alter bzw. mit Kinderwunsch, die bereits Psychopharmaka einnehmen. Und andere Frauen erkranken erstmalig psychisch, während sie schwanger sind. Daher ist die Frage nach Psychopharmaka in der Schwangerschaft ein Problem, mit dem man im klinischen Alltag immer häufiger konfrontiert wird.

Bei Frauen in gebärfähigen Alter sollte daher schon bei der Einstellung einer psychopharmakologischen Medikation darauf geachtet werden, dass keine Substanzen mit einem hohen Risiko für Embryotoxizität gegeben werden. Insbesondere für den Fall einer ungeplanten Schwangerschaft und aber auch, damit nicht wegen Kinderwunsches eine bestehende und wirksame Medikation gewechselt werden muss. Was es bei der Psychopharmakagabe in der Schwangerschaft generell zu beachten gilt, sind die potenziellen negativen Folgen oder unerwünschte Wirkungen, die zum einen in der Frühschwangerschaft, also im ersten Trimenon auftreten können. Hierbei handelt es sich primär um ein erhöhtes Risiko für Fehlbildungen. Medikamente, die zwar kein erhöhtes Fehlbildungsrisiko haben, könnten aber trotzdem zu Schwangerschafts- oder Geburtskomplikationen führen, wenn sie im zweiten und dritten Trimenon bzw. bis zur Geburt gegeben werden. Bei allen Studien, die es mittlerweile gibt, muss man aber immer im Blick behalten, dass es aus ethischen Gründen keine randomisiert-kontrollierten, doppelt verblindeten Studien im Bereich Psychopharmakologie und Schwangerschaft gibt und dass es viele Risikofaktoren für Schwangerschafts- und Geburtskomplikationen sowie die spätere Entwicklung der Kinder gibt, die ebenfalls einen starken Einfluss haben können und die häufig nicht in Studien miteinbezogen werden wie Nikotin-, Alkohol- und illegaler Drogenkonsum, BMI der Mutter, somatische
Komorbiditäten, genetische Faktoren und nicht zuletzt die psychische Erkrankung der Mutter selbst [1]. Auch schon deswegen sollte die Entscheidung für oder gegen die Gabe einer Medikation in der Schwangerschaft immer individuell unter Einbeziehung der Krankheitsanamnese und biografischer Faktoren unter RisikoNutzen-Analyse mit den Eltern gemeinsam getroffen werden. Die Kenntnis der spezifischen Risiken der verschiedenen Medikamente ist unerlässlich, spiegelt aber natürlich auch immer nur den aktuellen Stand der Wissenschaft wider, und in vielen Bereichen fehlen noch Studien. Diese SOP gibt einen Überblick über das Vorgehen bei geplanter und ungeplanter Schwangerschaft ( $\triangleright$ Abb. 1) und zusätzlich werden die aus unserer Sicht häufigsten Psychopharmaka bezüglich des Risikos für Fehlbildungen evaluiert sowie Risiken der Medikamentengabe im zweiten und dritten Trimenon geschildert. Auf spezifische Medikamente in der Stillzeit kann in diesem Artikel nicht eingegangen werden.

\section{Psychopharmakotherapie im ersten Trimenon}

\section{Relativ hohes Fehlbildungsrisiko}

Valproat

Aufgrund des erwiesenermaßen hohen gesamten Fehlbildungsrisikos von ungefähr $10 \%$, welches unter anderem spinale Fehlbildungen, aber auch kardiale Fehlbildungen beinhaltet sowie eines negativen Effektes auf IQ und psychomotorische Entwicklung bei den exponierten Kindern sollte Valproat nicht in der Schwangerschaft bzw. bei Frauen im gebärfähigen Alter gegeben werden [2].

\section{Carbamazepin/Oxcarbazepin}

Carbamazepin (CBZ) wird ein signifikant erhöhtes Risiko für Neuralrohrdefekte (bis zu $1 \%$ ) zugeschrieben, ebenso für kraniofaziale Defekte (bis zu 11\%) und Entwicklungsverzögerungen bei exponierten Kindern (bis 20\%) [2,3]. Oxcarbazepin, was gelegentlich auch zur Behandlung der bipolaren Störung eingesetzt wird, hat wohl ein deutlich geringeres Fehlbildungsrisiko, die Datenlage ist hier aber noch zu spärlich, um den Einsatz in der Schwangerschaft zu empfehlen [3].

\section{Topiramat}

Topiramat wurde mit einem höheren Fehlbildungsrisiko als Lamotrigin in Verbindung gebracht [2].

\section{Relativ geringes Fehlbildungsrisiko} Lithium

In neueren Studien konnte gezeigt werden, dass das Risiko, insbesondere für schwere kardiale Fehlbildungen, deutlich geringer ist als früher gedacht. Auch konnten in wenigen Studien keine negativen Effekte auf die weitere Entwicklung der Kinder gezeigt werden, hier ist die Datenlage allerdings noch recht spärlich. Dem gegenüber steht das fast $70 \%$ ige Rückfallrisiko postpartaler Krankheitsepisoden bei bipolaren Patientinnen. Daher wird heutzutage in vielen Fällen eher davon abgeraten, Lithium abzusetzen bei einer geplanten Schwangerschaft [4]. Allerdings konnte auch gezeigt werden, dass das Fehlbildungsrisiko höher ist, je höher die Lithiumkonzentration im Serum ist. Zudem steigt die renale Lithiumelimination während der Schwangerschaft stetig an, was zu fallenden Lithiumspiegeln führt. Nach Entbindung steigt die Lithiumkonzentration dann wieder rasch an, sodass eine Dosisreduktion erfolgen muss, um Intoxikationen vermeiden. Zusammenfassend kann Lithium während der Schwangerschaft nach Aufklärung über ein potenzielles, wenn auch geringes kardiales Fehlbildungsrisiko weiterverordnet werden. Allerdings müssen häufige Spiegelkontrollen sowie Nierenwert- und Schilddrüsenkontrollen erfolgen und die Lithiumgabe sollte über 2-3 Einzeldosierungen über den Tag erfolgen [5]. 


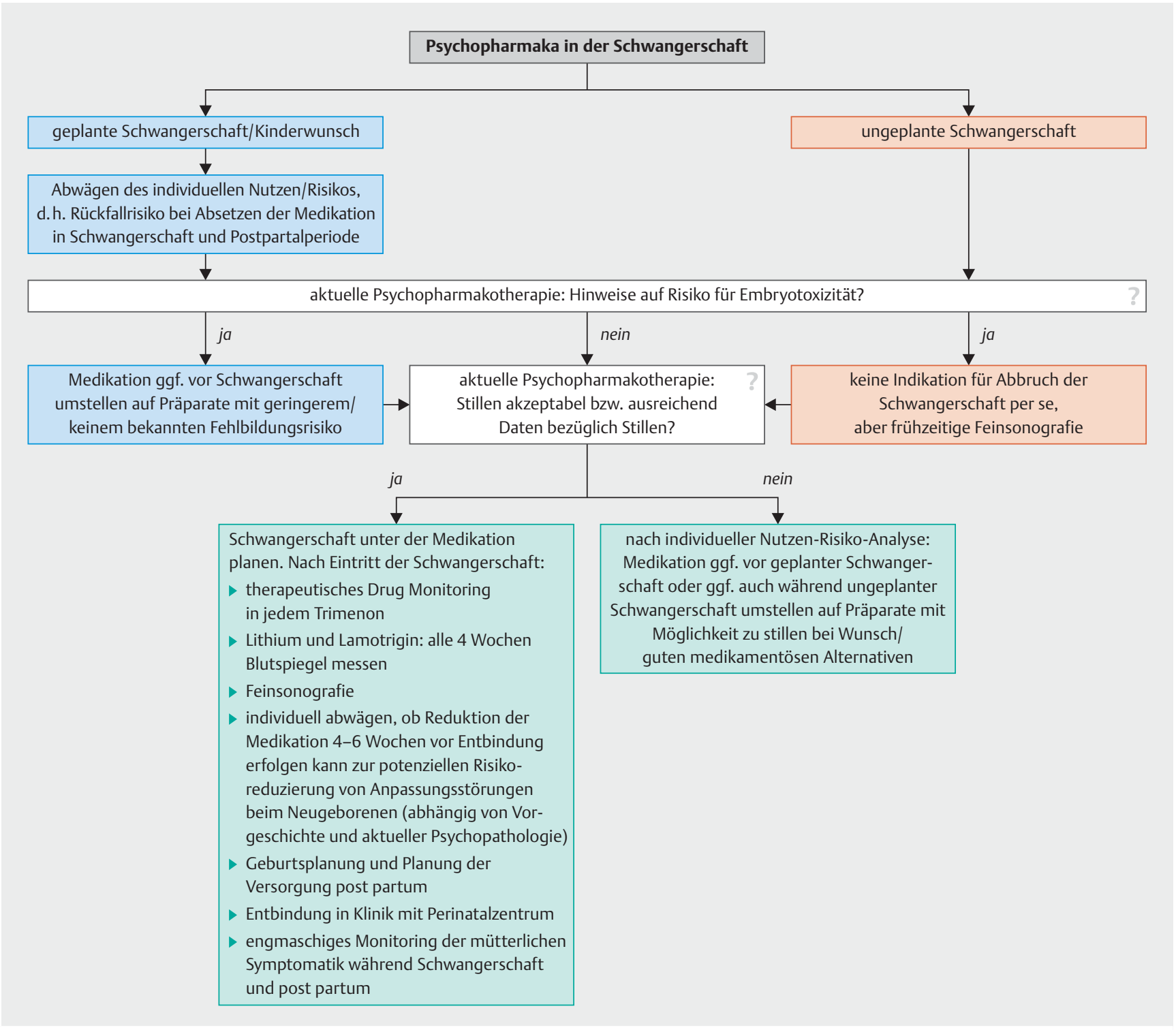

- Abb. 1 Psychopharmaka-Gabe in der Schwangerschaft.

\section{Lamotrigin}

In einigen Studien konnte kein erhöhtes Fehlbildungsrisiko bei Kindern gefunden werden, die mit Lamotrigin (LTG) exponiert worden waren [3], in anderen Studien nur ein sehr leicht erhöhtes generelles Malformationsrisiko [5,6]. Es wurden bisher keine negativen Effekte auf die kognitive Entwicklung von exponierten Kindern berichtet. Eine kürzliche Studie fand Hinweise auf ein erhöhtes Risiko für Autismus-Spektrum-Störungen, aber dieser Befund sollte erst noch mit einem geeigneten Studiendesign überprüft werden [7].
Die Besonderheit, die es bei LamotriginGabe in der Schwangerschaft zu beachten gibt, ist, dass der Abbau von Lamotrigin stark von Östrogenspiegeln abhängig ist. Das heißt, dass im Verlaufe der Schwangerschaft der LTG-Spiegel sinkt und die Dosis entsprechend erhöht werden muss, ca. 3-4 Wochen nach Entbindung steigt der Lamotrigin-Spiegel dann wieder an, sodass die eingenommene Menge wieder verringert werden muss [8].

\section{Pregabalin}

Zunächst gab es Hinweise, dass das Malformationsrisiko unter Pregabalin-Therapie erhöht sein könnte, mittlerweile wurde aber eine Kohortenstudie veröffentlich, die das nicht belegen konnte. Letztendlich gibt es aber noch zu wenige Daten, um eine endgültige Aussage treffen zu können $[9,10]$.

\section{Risperidon und Paliperidon}

Huybrechts und Kollegen fanden ein kleines, aber signifikantes Risiko für alle, insbesondere kardiale Fehlbildungen assoziiert mit Risperidon [11]. Diese Risikoerhöhung ist allerdings sehr klein und es ist auch nach wie vor unklar, ob es wirklich einen kausalen Zusammenhang gibt. Dennoch sollte man, wenn möglich, im ersten Trimenon auf Olanzapin oder Quetiapin 
ausweichen. Daten für Paliperidon bezüglich Einnahme in der Schwangerschaft gibt es keine.

\section{Benzodiazepine und Z-Substanzen}

Benzodiazepin-Medikation sowie Zolpidem und Zopiclon während der Schwangerschaft führten in mehreren Studien zu keiner relevanten Erhöhung für generelle Malformationen [12-14]. Allerdings wurde in anderen Studien ein leicht erhöhtes Risiko für Lippen-Kiefer-Gaumen-Spalten diskutiert. Benzodiazepine und auch Zopiclon und Zolpidem überwinden die Plazenta und können im Embryo/Fetus akkumulieren [15]. Postnatal kann es daher auch bei Benzodiazepin-Gabe häufiger zu Anpassungsstörungen beim Kind kommen, insbesondere „floppy-infant“-Syndrom und Atemstörungen können auftreten [16]. Auch gibt es Hinweise, dass Benzodiazepine in der späteren Schwangerschaft mit Frühgeburtlichkeit und zu großen und zu kleinen Kindern assoziiert sind [15].

Potenziell Paroxetin, Fluoxetin sowie fraglich Clomipramin (siehe unten).

\section{Kein bekanntes Fehlbildungsrisiko}

\section{Quetiapin}

Die Datenlage ist mittlerweile ausreichend, um sagen zu können, dass Quetiapin nicht mit einem erhöhten Fehlbildungsrisiko assoziiert ist [17]. Was während der Gabe in der Schwangerschaft zu beachten ist, ist, dass die QuetiapinSerumspiegel im Laufe der Schwangerschaft unter gleichbleibender Dosierung abfallen und nach Entbindung nach wenigen Tagen wieder ansteigen. Daher kann es sein, dass man während der Schwangerschaft und nach Entbindung die Dosierung unter engmaschiger Kontrolle zum einen der Psychopathologie und unerwünschten Wirkungen wie einer übermäßigen Sedierung der Mutter, zum anderen ggf. mithilfe eines therapeutischen Drug Monitorings anpassen muss [18].

\section{Aripiprazol}

Zunächst gab es Bedenken wegen Tierversuchen, die Hinweise für teratogene Effekte bei Exposition mit Aripipazol in der Schwangerschaft zeigten. Mittlerweile gibt es jedoch ausreichend humane Da- ten, die keine Hinweise für ein erhöhtes Malformationsrisiko zeigen $[11,19]$.

\section{Olanzapin}

Es gibt keine Hinweise für ein erhöhtes Fehlbildungsrisiko nach Olanzapin-Exposition im ersten Trimester. Es liegen jedoch Studien vor, die zeigen, dass möglicherweise ein erhöhtes Risiko für Schwangerschaftsdiabetes bei mit Olanzapin behandelten Schwangeren besteht, die Datenlage ist aber nicht ganz konsistent [11, 19].

\section{Clozapin}

Von den bisher publizierten Daten ausgehend, sieht es nicht so aus, als wäre Clozapin mit einem erhöhten Risiko für Fehlbildungen assoziiert. Allerdings gibt es Hinweise darauf, dass Clozapin-Einnahme in der späteren Schwangerschaft mit einem erhöhten Risiko für erhöhtes Geburtsgewicht bei den Kindern einhergehen könnte und möglicherweise auch mit schwereren Anpassungsstörungen bei den Neugeborenen, als bei den anderen Antipsychotika berichtet wurden. Allerdings ist die Datenlage hierzu recht spärlich. Dennoch sollten, wenn möglich, in der Schwangerschaft andere Antipsychotika mit einem besseren Sicherheitsprofil verwendet werden [19].

Haloperidol und Promethazin als Vertreter der älteren, typischen Antipsychotika sind beide relativ gut untersucht und scheinen kein erhöhtes Fehlbildungsrisiko zu haben [11].

\section{Stimulanzien und Atomoxetin}

In den bisherigen Studien gibt es keine Hinweise auf ein erhöhtes Fehlbildungsrisiko bei Einnahme von ADHS-Medikation im ersten Trimenon [20-22].

Antidepressiva (außer Paroxetin, Fluoxetin, ggf. Clompramin)

In einer großen populationsbasierten Studie konnten, nach Adjustierung von verschiedenen Risikofaktoren, keine signifikanten Risikoerhöhungen für kardiale Malformationen bei SSRI-Exposition im ersten Trimester gefunden werden [23], Ausnahmen sind Paroxetin und Fluoxetin, wobei das nicht konsistent berichtet wird [24]. Bezüglich der trizyklischen Antidepressiva wurden in anderen Studien nur bei Clomi- pramin stärkere Hinweise auf Risikoerhöhung bezüglich angeborener kardialer Defekte gesehen und auch postpartale Komplikationen (siehe unten) könnten bei Clomipramin stärker ausgeprägt sein [25]. Zu Mirtazapin, Bupropion, Johanniskraut gibt es noch etwas weniger Befunde, allerdings konnten bisher ebenfalls keine fruchtschädigenden Effekte gefunden werden, sodass der Einsatz in der Schwangerschaft als vertretbar erscheint [26-29].

\section{Unzureichende Datenlage}

Bei den neueren Antidepressiva und Antipsychotika wie Ziprasidon, Asenapin, Cariprazin, Milnacipran, Agomelatin sowie älteren Antipsychotika wie Pipamperon, Melperon, Chlorprothixen und Prothipendyl sind nicht ausreichend humane Daten für eine Risikoabschätzung vorhanden.

\section{Psychopharmakotherapie im zweiten und dritten \\ Trimenon}

Eine sehr seltene, aber schwerwiegende Komplikation bei Neugeborenen, die vor allen Dingen mit SSRI-Exposition in der späteren Schwangerschaft in Verbindung gebracht wird, ist persistierender Lungenhochdruck (persistierende pulmonale Hypertonie). Huybrechts und Kollegen fanden ein kleines, aber signifikant erhöhtes Risiko für diese Erkrankung bei einer SSRIExposition im letzten Trimenon [30]. Zudem werden immer wieder erhöhte Risiken für Frühgeburt und niedriges Geburtsgewicht in Verbindung mit Psychopharmaka gebracht [31]. Eine neuere Studie und eine kürzlich publizierte Metaanalyse geben allerdings Hinweise, dass die erhöhten Raten an Frühgeburt und geringem Geburtsgewicht eher mit z.B. der depressiven Erkrankung der Mutter an sich als mit der Medikation zusammenhängen könnten $[32,33]$. Aber es wurden auch negative Effekte der SSRI-Exposition auf das Arousal und Selbstregulation sowie Bewegungsqualität bei den Kindern während des ersten Lebensmonats gefunden im Vergleich zu Kindern nicht behandelter Müttern mit einer Depression in der Vorgeschichte und gesunden Kontrollen [34]. Zudem gab es in dieser Studie eine kleinere Gruppe, die mit Benzodiazepinen 
und SSRIs in Kombination behandelt wurde, was die Symptome bei den Kindern wie erwartet verstärkte.

Schwerwiegende negative Einflüsse auf die spätere Entwicklung der Kinder (über das 3. Lebensjahr hinaus) wurden bei Psychopharmakaeinnahme in der Schwangerschaft bisher nicht beschrieben, außer in wenigen Studien, die auf eine fragliche Assoziation von Autismus-Spektrum-Störungen und SSRI-Exposition in der Schwangerschaft hinwiesen $[35,36]$. In einer neuen Metaanalyse wird angezweifelt, dass die SSRI-Exposition in der Schwangerschaft zu einem erhöhten Risiko für Autismus-Spektrum-Störungen führt [37].

Das Risiko für Anpassungsstörungen (z. B. Übererregbarkeit, Tremor, erhöhter oder erniedrigter Muskeltonus, Trinkstörungen, Atemnotsyndrom, Hypoglykämie, auffälliges Schlafverhalten mit vermehrten Schreckreaktionen und verlängerten REMPhasen) wird bei bis zu 2 bis 3 von 10 der Psychopharmaka-exponierten Kinder angegeben, unabhängig von der Substanzklasse [38]; daher sollten die Mütter in einer Geburtsklinik mit angeschlossenem Perinatalzentrum entbinden. In der Regel sind die Anpassungsstörungen jedoch eher leicht ausgeprägt und nach kurzer Zeit selbstlimitierend [39].

\section{EXKURS}

\section{Psychopharmaka in der Stillzeit}

Die ist nochmal ein ganz eigenes Thema, worauf im Rahmen dieses Artikels nicht eingegangen werden kann. Was man aber beachten sollte: Wenn ein Kind schon in der Schwangerschaft mit Medikamenten exponiert war, welche auch über das Nabelschnurblut in das Kind gelangen können, dann sollte nicht automatisch von Stillen abgeraten werden. Im Gegenteil gibt es sogar Studien, die zeigen, dass etwaige Anpassungsstörungen bei gestillten Kindern geringer waren, und zudem kommt bei den meisten Medikamenten auch weniger über die Muttermilch in den Kindern an, als es vorher über die
Nabelschnur geschehen ist [40]. Auch hier gibt es aber Medikamente, die eher empfohlen werden können als andere und zudem muss wieder eine individuelle Nutzen-RisikoAnalyse mit der Mutter bzw. beiden Elternteilen erfolgen.

\section{FAZIT}

Die Gabe von Psychopharmaka während der Schwangerschaft sollte immer nach individueller NutzenRisiko-Abwägung getroffen werden mit beiden Elternteilen. Es gibt nur sehr wenige Medikamente, die stark fruchtschädigend wirken, die meisten Psychopharmaka haben nur ein leichtes oder gar kein bekanntes Fehlbildungsrisiko. Anpassungsstörungen können bei allen Psychopharmaka auftreten, in der Regel sind diese aber selbstlimitierend und nicht vital bedrohlich für das Kind.

Zum Schnellen Nachschauen eignet sich www.embryotox.de.

Interessenkonflikt

Die Autorin ist als Beraterin und Autorin tätig für Shire und Medice Arzneimittel Pütter GmbH.

\section{Autorinnen/Autoren}

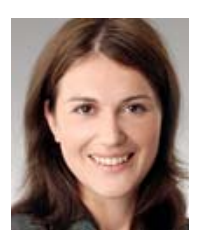

Sarah Kittel-Schneider

PD Dr. med. Sarah KittelSchneider, Studium der Humanmedizin 2001-2008, Würzburg. 2014 Fachärztin für Psychotherapie und Psychotherapie und Oberärztin, Uniklinik Würzburg. 2014-2017 leitende Oberärztin, Uniklinik Frankfurt. 2017 stellvertretende Klinikdirektorin, Uniklinik Frankfurt. 2018 Habilitation. Schwerpunkte: affektive Erkrankungen, ADHS, peripartale Psychiatrie, fluide Biomarker und Zellmodelle psychiatrischer Erkrankungen.

\section{Korrespondenzadresse}

PD Dr. med. Sarah Kittel-Schneider Klinik für Psychiatrie, Psychotherapie und Psychosomatik

Universitätsklinikum Frankfurt,

Goethe Universität

Heinrich-Hoffmann-Str. 10

60528 Frankfurt

sarah.kittel-schneider@kgu.de

\section{Erstveröffentlichung}

Dieser Beitrag wurde erstveröffentlicht in: PSYCH up2date 2019; 13: 100-105

Literatur

[1] Herba CM, Glover V, Ramchandani PG et al. Maternal depression and mental health in early childhood: an examination of underlying mechanisms in low-income and middleincome countries. Lancet Psychiatry 2016; 3: 983-992. doi:10.1016/S2215-0366(16) 30148-1

[2] Andrade C. Major Congenital Malformations Associated With Exposure to Antiepileptic Drugs During Pregnancy. J Clin Psychiatry 2018; 79. doi:10.4088/JCP.18f12449

[3] Tomson T, Battino D, Bonizzoni E et al. Comparative risk of major congenital malformations with eight different antiepileptic drugs: a prospective cohort study of the EURAP registry. Lancet Neurol 2018; 17: 530-538. doi:10.1016/S1474-4422(18)30107-8

[4] Diav-Citrin O, Shechtman S, Tahover E et al. Pregnancy outcome following in utero exposure to lithium: a prospective, comparative, observational study. Am J Psychiatry 2014; 171: 785-794. doi:10.1176/appi.ajp.2014. 12111402

[5] Patorno E, Huybrechts KF, Bateman BT et al. Lithium Use in Pregnancy and the Risk of Cardiac Malformations. N Engl J Med 2017; 376: 2245-2254. doi:10.1056/NEJMoa 1612222

[6] Dolk $\mathrm{H}$, Wang $\mathrm{H}$, Loane $\mathrm{M}$ et al. Lamotrigine use in pregnancy and risk of orofacial cleft and other congenital anomalies. Neurology 2016; 86: 1716-1725. doi:10.1212/WNL. 0000000000002540

[7] Veroniki AA, Rios P, Cogo E et al. Comparative safety of antiepileptic drugs for neurological development in children exposed during pregnancy and breast feeding: a systematic review and network meta-analysis. BMJ Open 2017; 7: e017248. doi:10.1136/ bmjopen-2017-017248

[8] Clark CT, Klein AM, Perel JM et al. Lamotrigine dosing for pregnant patients with bipolar disorder. Am J Psychiatry 2013; 170 : 1240-1247. doi:10.1176/appi.ajp.2013. 13010006 
[9] Patorno E, Bateman BT, Huybrechts KF et al. Pregabalin use early in pregnancy and the risk of major congenital malformations. Neurology 2017; 88: 2020-2025. doi:10.1212/ WNL.0000000000003959

[10] Winterfeld U, Merlob P, Baud D et al. Pregnancy outcome following maternal exposure to pregabalin may call for concern. Neurology 2016; 86: 2251-2257. doi:10.1212/ WNL.0000000000002767

[11] Huybrechts KF, Hernandez-Diaz S, Patorno E et al. Antipsychotic Use in Pregnancy and the Risk for Congenital Malformations. JAMA Psychiatry 2016; 73: 938-946. doi:10.1001/ jamapsychiatry.2016.1520

[12] Wikner BN, Kallen B. Are hypnotic benzodiazepine receptor agonists teratogenic in humans? J Clin Psychopharmacol 2011; 31: 356-359. doi:10.1097/JCP.0b013e3182197 055

[13] Enato E, Moretti M, Koren G. The fetal safety of benzodiazepines: an updated meta-analysis. J Obstet Gynaecol Can 2011; 33: 46-48

[14] Ban L, West J, Gibson JE et al. First trimester exposure to anxiolytic and hypnotic drugs and the risks of major congenital anomalies: a United Kingdom population-based cohort study. PLoS One 2014; 9: e100996. doi:10.1371/journal.pone.0100996

[15] Okun ML, Ebert R, Saini B. A review of sleeppromoting medications used in pregnancy. Am J Obstet Gynecol 2015; 212: 428-441. doi:10.1016/j.ajog.2014.10.1106

[16] Uzun S, Kozumplik O, Jakovljevic M et al. Side effects of treatment with benzodiazepines. Psychiatr Danub 2010; 22: 90-93

[17] Cohen LS, Goez-Mogollon L, Sosinsky AZ et al. Risk of Major Malformations in Infants Following First-Trimester Exposure to Quetiapine. Am J Psychiatry 2018. doi:10.1176/ appi.ajp.2018.18010098

[18] Uguz F. Prophylactic use of olanzapine and quetiapine from pregnancy to the postpartum period in women with bipolar disorder: a case series. J Matern Fetal Neonatal Med 2017; 30: 2569-2571. doi:10.1080/ 14767058.2016.1256991

[19] Damkier P, Videbech P. The Safety of SecondGeneration Antipsychotics During Pregnancy: A Clinically Focused Review. CNS Drugs 2018; 32: 351-366. doi:10.1007/s40263018-0517-5

[20] Norby U, Winbladh B, Kallen K. Perinatal Outcomes After Treatment With ADHD Medication During Pregnancy. Pediatrics 2017. doi:10.1542/peds.2017-0747

[21] Diav-Citrin O, Shechtman S, Arnon J et al. Methylphenidate in Pregnancy: A Multicenter, Prospective, Comparative, Observational Study. J Clin Psychiatry 2016; 77: 11761181. doi:10.4088/JCP.15m10083
[22] Dideriksen D, Pottegard A, Hallas ] et al. First trimester in utero exposure to methylphenidate. Basic Clin Pharmacol Toxicol 2013; 112: 73-76. doi:10.1111/bcpt.12034

[23] Huybrechts KF, Hernandez-Diaz S, Avorn J. Antidepressant use in pregnancy and the risk of cardiac defects. N Engl J Med 2014; 371: 1168-1169. doi:10.1056/NEJMc1409203

[24] Womersley K, Ripullone K, Agius M. What are the risks associated with different Selective Serotonin Re-uptake Inhibitors (SSRIs) to treat depression and anxiety in pregnancy? An evaluation of current evidence. Psychiatr Danub 2017; 29: 629-644

[25] Gentile S. Tricyclic antidepressants in pregnancy and puerperium. Expert Opin Drug Saf 2014; 13: 207-225. doi:10.1517| 14740338.2014.869582

[26] Smit M, Dolman KM, Honig A. Mirtazapine in pregnancy and lactation - A systematic review. Eur Neuropsychopharmacol 2016; 26 : 126-135. doi:10.1016/j.euroneuro.2015. 06.014

[27] Winterfeld $U$, Klinger $G$, Panchaud $A$ et al. Pregnancy outcome following maternal exposure to mirtazapine: a multicenter, prospective study. I Clin Psychopharmacol 2015; 35: 250-259. doi:10.1097/JCP. 0000000000000309

[28] Nanovskaya TN, Oncken C, Fokina VM et al. Bupropion sustained release for pregnant smokers: a randomized, placebo-controlled trial. Am J Obstet Gynecol 2017; 216: 420. e421-422.e429. doi:10.1016/j.ajog.2016. 11.1036

[29] Kolding L, Pedersen LH, Henriksen TB et al. Hypericum perforatum use during pregnancy and pregnancy outcome. Reprod Toxicol 2015; 58: 234-237. doi:10.1016/ j.reprotox.2015.10.003

[30] Huybrechts KF, Bateman BT, Palmsten K et al. Antidepressant use late in pregnancy and risk of persistent pulmonary hypertension of the newborn. JAMA 2015; 313: 2142-2151. doi:10.1001/jama.2015.5605

[31] Ross LE, Grigoriadis S, Mamisashvili L et al. Selected pregnancy and delivery outcomes after exposure to antidepressant medication: a systematic review and meta-analysis. JAMA Psychiatry 2013; 70: 436-443. doi:10.1001/jamapsychiatry.2013.684

[32] Cantarutti A, Merlino L, Monzani E et al. Is the Risk of Preterm Birth and Low Birth Weight Affected by the Use of Antidepressant Agents during Pregnancy? A Population-Based Investigation. PLoS One 2016; 11: e0168115. doi:10.1371/journal.pone. 0168115
[33] Jarde A, Morais M, Kingston D et al. Neonatal Outcomes in Women With Untreated Antenatal Depression Compared With Women Without Depression: A Systematic Review and Meta-analysis. JAMA Psychiatry 2016 73: 826-837. doi:10.1001/jamapsychiatry. 2016.0934

[34] Salisbury AL, O'Grady KE, Battle CL et al. The Roles of Maternal Depression, Serotonin Reuptake Inhibitor Treatment, and Concomitant Benzodiazepine Use on Infant Neurobehavioral Functioning Over the First Postnatal Month. Am J Psychiatry 2016; 173: 147-157. doi:10.1176/appi.ajp.2015.14080989

[35] El Marroun H, White TJ, van der Knaap NJ et al. Prenatal exposure to selective serotonin reuptake inhibitors and social responsiveness symptoms of autism: population-based study of young children. Br J Psychiatry 2014; 205: 95-102. doi:10.1192/bjp.bp.113. 127746

[36] Boukhris T, Sheehy O, Mottron L et al. Antidepressant Use During Pregnancy and the Risk of Autism Spectrum Disorder in Children. JAMA Pediatr 2016; 170: 117-124. doi:10.1001/jamapediatrics.2015.3356

[37] Brown HK, Hussain-Shamsy N, Lunsky Y et al. The Association Between Antenatal Exposure to Selective Serotonin Reuptake Inhibitors and Autism: A Systematic Review and Meta-Analysis. I Clin Psychiatry 2017; 78: e48-e58. doi:10.4088/JCP.15r10194

[38] Kieviet N, van Keulen V, van de Ven PM et al. Serotonin and poor neonatal adaptation after antidepressant exposure in utero. Acta Neuropsychiatr 2017; 29: 43-53. doi:10.1017/neu.2016.30

[39] Forsberg L, Naver L, Gustafsson LL et al. Neonatal adaptation in infants prenatally exposed to antidepressants-clinical monitoring using Neonatal Abstinence Score. PLoS One 2014; 9: e111327. doi:10.1371/journal. pone. 0111327

[40] Smit M, Wennink H, Heres M et al. Mirtazapine in pregnancy and lactation: data from a case series. J Clin Psychopharmacol 2015; 35: 163-167. doi:10.1097/JCP.0000000000000 279

\section{Bibliografie}

DOI https://doi.org/10.1055/a-0915-5635 Geburtsh Frauenheilk 2020; 80: 35-39 @ Georg Thieme Verlag KG Stuttgart . New York I ISSN 0016-5751 